\title{
CISTICERCOSE HUMANA DIAGNOSTICADA EM HOSPITAL GERAL, SÃO PAULO, SP (BRASIL)
}

\author{
Aluízio de Barros Barreto Machado* \\ Carmen Silvia de Melo Pialarissi** \\ Adelaide José Vaz**
}

MACHADO, A. de B. B. et al. Cisticercose humana diagnosticada em hospital geral, Săo Paulo, SP (Brasil). Rev.Saúde públ., S.Paulo, 22: 240 - 4, 1988.

RESUMO: Foi realizado estudo retrospectivo de pacientes internados no Hospital das Clínicas da Faculdade de Medicina da Universidade de São Paulo, São Paulo (Brasil), no periodo de 7 anos (1979 a 1985), tendo sido diagnosticados 260 casos de cisticercose, correspondendo a $0,2 \%$ do total estudado. Os dados obtidos mostraram uma distribuição aproximadamente igual quanto ao sexo e o acometimento maior na faixa etária de 20 a 50 anos $(53,8 \%$ ). A neurocisticercose foi a forma mais freqüente e seu elevado percentual $(91,5 \%)$ foi atribuído às características da população estudada. Apesar do recente desenvolvimento da tecnologia diagnóstica e terapêutica específicas, o tempo de permanência hospitalar médio foi de 35 dias e a letalidade média de $14,6 \%$, apontando para a necessidade de medidas profiláticas através de programas de saúde pública.

UNITERMOS: Cisticercose, incidência. Epidemiologia. Hospitalização.

\section{INTRODUÇÃO}

Estudos epidemiológicos efetuados em países em desenvolvimento, onde predominam os climas tropical e subtropical, tếm mostrado que as condiçoes sócio-econômicas favorecem a proliferação dè doença de grande importância à Saúde Pública. ${ }^{7,12,14}$

A cisticercose humana, doença causada pelo Cysticercus cellulosae, larva da Taenia solium, agrupa-se entre aquelas entidades, pela elevada incidência, pela gravidade dos quadros clínicos e pela precariedade dos recursos terapêuticos específicos. ${ }^{217}$

Programas de Saúde Pública direcionados a obter a redução da incidência do complexo teníase-cisticercose têm sido adotados com sucesso em alguns países europeus. ${ }^{8,12}$

Apesar das dificuldades de ordem biológica, estatística e técnica inerentes a uma investigação epidemiológica ${ }^{19,20}$, relatos fracionários diversos levam à conclusăo de que a cisticercose humana tem distribuição geográfica mundial, sendo freqüente na Ásia, Europa, África e, principalmente, na América Latina. ${ }^{12,19}$

México, Peru, Chile e Brasil têm sido apontados como os países latino-americanos onde é maior a incidência de pacientes internados por neurocisticercose ${ }^{16}$, a forma mais comum e grave da doença que reflete, aproximadamente, a morbidade da cisticercose humana ${ }^{15}$.

No Brasil, alguns estudos sobre neurocisticercose realizados em serviços especializados em Neurologia de Såo Paulo,18,20 têm fornecido subsídios relevantes à investigação de aspectos epidemiológicos da cisticercose. Carecem estes estudos, pelas dificuldades já citadas, de informaçoes sobre as demais formas da cisticercose humana, as quais permitiriam um dimensionamento mais adequado do problema sanitário. Por outro lado, a atualização contínua destes estudos é contribuiçăo necessária à vigilância epidemiologica, uma das principais medidas que visam à erradicação da cisticercose humana. ${ }^{4.8}$

O objetivo do presente trabalho é apresentar alguns aspectos epidemiológicos atuais da cisticercose humana em Săo Paulo, obtidos em pacientes internados.

\section{MATERIAL E MÉTODOS}

De 126.968 pacientes internados no Hospital das Clínicas da Faculdade de Medicina da Universidade de São Paulo (HCFMUSP), no período de $1^{2}$ de janeiro de 1979 a 31 de dezembro de 1985 ,

- Seção de Líquido Cefalorraqueano da Divisão de Laboratório Central do Hospital das Clínicas da Faculdade de Medicina da Universidade de São Paulo - Av. Dr. Enéas Carvalho de Aguiar, s/ n - 01246 - São Paulo, SP Brasil.

* Seção de Sorologia da Divisão de Biologia Médica do Instituto Adolfo Lutz - Av. Dr. Arnaldo, $351-10^{\circ}$ andar - 01246 - São Paulo , SP - Brasil. 
excluídas as reinternaçסes, foi feito diagnóstico de cisticercose em $260(0,2 \%)$. Esse diagnóstico foi feito com base nos criterios clínicos, radiológicos e imunológicos $3,10,18$ adotados pelo HCFMUSP. Em alguns casos a cirugia e a autópsia confirmaram o diagnóstico.

Os seguintes dados foram obtidos: incidência, distribuiçăo por idade e sexo, distribuiçăo por diagnóstico clínico-topográfico, distribuição por tempo de permanência hospitalar, letalidade.

\section{RESULTADOS}

$A$ incidência da cisticercose em pacientes internados foi estudada através de freqüência percentual de internaçס̃es, obtida a partir dos dados apresentados na Tabela 1.

As Tabelas 2, 3, 4 e 5 mostram, respectivamente, a distribuição dos 260 casos com diagnóstico de cisticercose, segundo sexo/idade; diagnóstico clínico topográfico; tempo de permanência hospitalar; $e$ letalidade.

\section{DISCUSSÃO}

A incidência da neurocisticercose em pacientes atendidos em serviços de Neurologia de São Paulo tem sido objeto de algumas publicaçōes que apontam, no período de 1939 a 1979, para uma percentagem de 0,31 a 2,98 dos atendimentos $1,2,9,17,20$. No entanto a literatura é pobre em citaçôes sobre a incidência de cisticercose em hospitais gerais. No HCFMUSP, Machado ${ }^{11}$ observou a incidência de $0,2 \%$ no período de 1969 a 1978. Os dados da Tabela 1, obtidos no período de 1979 a 1985, na mesma instituição, mostraram que a incidência média atualizada permanece rigorosamente igual. A freqüência anual de internaçōes foi bastante oscilante na série histórica apresentada, fato já assinalado por Spina-França ${ }^{17}$ para pacientes portadores de neurocisticercose no período de 1947 a 1955, indicando não haver tendência atual de redução da freqüência da doença entre os pacientes internados.

A caracterização da população estudada, quanto ao sexo, foi similar às descritas na literatu$\mathrm{ra}^{2,16,21}$ e apontou para uma distribuição aproximadamente igual: $50,4 \%$ do sexo masculino e $49,6 \%$ feminino. A idade por ocasião do diagnóstico variou entre 1 e 83 anos, sendo a cisticercose mais freqüente nas terceira, quarta e quinta décadas, confirmando as observaçoes de Schenone ${ }^{16}$ e Gobbi $i^{6}$ e sugerindo que as faixas etárias mais atingidas são as economicamente mais produtivas.

Dos 260 casos de cisticercose internados, 238 $(91,5 \%)$ tiveram diagnóstico de neurocisticercose, valor já esperado tendo em vista que a forma neurológica é a mais grave e, como apontam os

\section{TABELA 1}

Número de casos de cisticercose, número de internaçōes e freqüência (\%) de cisticercose. Hospital das Clínicas da Faculdade de Medicina da Universidade de São Paulo (1979 - 1985).

\begin{tabular}{cccc}
\hline Ano & $\begin{array}{c}\text { No de casos } \\
\text { Cisticercose }\end{array}$ & $\begin{array}{c}\text { Ne de Internados } \\
\text { em Geral }\end{array}$ & $\%$ \\
\hline 1979 & 25 & 17.795 & 0,14 \\
1980 & 32 & 19.999 & 0,16 \\
1981 & 41 & 19.104 & 0,21 \\
1982 & 34 & 15.117 & 0,22 \\
1983 & 33 & 19.272 & 0,17 \\
1984 & 55 & 19.005 & 0,29 \\
1985 & 40 & 16.676 & 0,24 \\
\hline Total & 260 & 126.968 & 0,20 \\
\hline
\end{tabular}

TABELA 2

Número e percentagem de pacientes com diagnóstico de cisticercose segundo sexo e faixa etária. Hospital das Clínicas da Faculdade de Medicina da Universidade de São Paulo (1979 - 1985)

\begin{tabular}{crrrrrr}
\hline \multirow{2}{*}{ Sexo } & \multicolumn{2}{c}{ Masculino } & \multicolumn{2}{c}{ Feminino } & \multicolumn{2}{c}{ Total } \\
\cline { 2 - 7 } Idade & $\mathrm{n}$ & $\%$ & $\mathrm{n}$ & $\%$ & $\mathrm{n}$ & $\%$ \\
\hline $1 \vdash 10$ & 10 & 3,8 & 18 & 7,0 & 28 & 10,8 \\
$10 \vdash 20$ & $\mathbf{1 6}$ & 6,2 & 17 & 6,5 & 33 & 12,7 \\
$20 \vdash 30$ & 23 & 8,8 & 30 & 11,5 & 53 & 20,3 \\
$30 \vdash 40$ & 28 & 10,8 & 23 & 8,8 & 51 & 19,7 \\
$40 \vdash 50$ & 19 & 7,3 & 17 & 6,5 & 36 & 13,8 \\
$50 \vdash 60$ & 15 & 5,8 & 14 & 5,5 & 29 & 11,2 \\
$60 \vdash 70$ & 10 & 3,8 & 5 & 1,9 & 15 & 5,7 \\
$70 \vdash 84$ & 2 & 0,8 & 0 & 0 & 2 & 0,8 \\
Ingnorada & 8 & 3,1 & 5 & 1,9 & 13 & 5,0 \\
\hline Total & 131 & 50,4 & 129 & 49,6 & 260 & 100,0 \\
\hline
\end{tabular}

\section{TABELA 3}

Número e percentagem de pacientes segundo diagnóstico clínico-topográfico. Hospital das Clínicas da Faculdade de Medicina da Universidade de São Paulo (1979 - 1985)

\begin{tabular}{lrr}
\hline Diagnóstico & No de Pacientes & $\%$ \\
\hline Neurodisticercose & 238 & 91,5 \\
Oftalmocisticercose & 7 & 2,7 \\
Cisticercose músculo-cutânea & 8 & 3,1 \\
Outros & 7 & 27 \\
\hline Total & 260 & 100,0 \\
\hline
\end{tabular}




\section{TABELA 4}

Número e percentagem de pacientes com cistcercose segundo tempo e permanência (dias). Hospital das Clínicas da Faculdade de Medicina da Universidade de Sao Paulo (1979 - 1985).

\begin{tabular}{rrr}
\hline \multicolumn{1}{c}{ Dias } & No de Padientes & $\%$ \\
\hline $1 \vdash 31$ & 146 & 56,2 \\
$31 \vdash 61$ & 84 & 32,3 \\
$61 \vdash 91$ & 11 & 4,2 \\
$91 \vdash 121$ & 7 & 2,7 \\
$121 \vdash 151$ & 7 & 2,7 \\
$151 \leftarrow 254$ & 5 & 1,9 \\
\hline Total & 260 & 100,0 \\
\hline
\end{tabular}

\section{TABELA 5}

Letalidade (\%) observada em 260 pacientes com cisticercose. Hospital das Clínicas da Faculdade de Medicina da Universidade de Såo Paulo (1979 - 1985).

\begin{tabular}{lccr}
\hline Ano & N $^{\circ}$ de 6bitos & N $^{0}$ de Casos & $\%$ \\
\hline 1979 & 3 & 25 & 12,0 \\
1980 & 3 & 32 & 9,4 \\
1981 & 6 & 41 & 14,6 \\
1982 & 8 & 34 & 23,5 \\
1983 & 10 & 33 & 30,3 \\
1984 & 5 & 40 & 9,1 \\
1985 & 3 & 260 & 7,5 \\
\hline Total & 38 & & 14,6 \\
\hline
\end{tabular}

levantamentos anatomopatológicos ${ }^{6,15}$ a mais freqüente. Considerando as características da populaçăo avaliada neste estudo, as demais formas de cisticercose năo diferem em valores relativos dos registrados na literatura ${ }^{16}$.

No presente trabalho năo foram inclứdas as reinternaçőes dos pacientes. Entretanto em cerca de metade dos casos o doente com neurocisticercose é internado mais de uma vez ${ }^{16}$. Estudos realizados no México, entre 1970 e 1972, revelaram que o custo de hospitalizaçăo de um paciente com diagnóstico de neurocisticercose é de cerca de US\$ $1.200^{13}$.

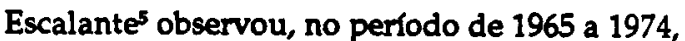
em 276 pacientes com neurocisticercose, um tempo de permanência hospitalar médio oscilando entre 42 e 46 dias. Em nossa populaçăo, o tempo de permanência médio foi de 35 dias, mediana de 28 dias, fato justificado pelo desenvolvimento da tecnologia diagnóstica e terapêutica obtido na última década às custas de novos investimentos.

Considerando o elevado número de casos assintomáticos que não sảo submetidos a critérios diagnósticos específicos, é difícil avaliar a letalidade por cisticercose ${ }^{15}$. Não há dúvidas, porém, de que a neurocisticercose é a responsável pela grande maioria dos óbitos, seja pela gravidade da mesma, seja em decorrência das complicaçóes do seu tratamento. A letalidade por neurocisticercose mostrada na literatura tem diminuído com o avanço recente das medidas terapêuticas adotadas. Segundo Schenone ${ }^{16}$, até 1950 era de $63,6 \%$ (em 110 casos), caindo para 32,7\% (em 905 casos) nas três últimas décadas. Em Săo Paulo, as principais publicaçōes mostram índices de 14,9\% (em 47 casos) $17,25,9 \%$ (em 166 casos) ${ }^{2}$ e $18 \%$ (em 233 casos) ${ }^{20}$. Em nossa amostragem a taxa média foi de $14,6 \%$ (260 casos) para cisticercose, valor que está de acordo com os acima referidos, tendo em vista que a maioria dos casos $(91,5 \%)$ tinha diagnóstico de neurocisticercose. Esta observaçăo mostra ainda que persiste elevada letalidade por esta moléstia.

Os aspectos epidemiologicos apresentados indicam que a cisticercose continua tendo representação nosologica importante na populaçăo estudada, tanto pela sua letalidade como pelo seu alto custo sócio-econômico.

Respeitadas as limitaçōes deste trabalho que, nos moldes de outras publicaçōes sobre o tema; incluiu apenas o estudo de formas mais graves que motivaram a internaçāo dos casos, é possivel concluir que a cisticercose humana mantém-se endêmica, provavelmente porque a carência de investimentos inviabiliza a execuçăo adequada dos Programas de Saúde Pública.

\section{AGRADECIMENTOS}

A Divisão de Arquivo Médico do Hospital das Clínicas da Faculdade de Medicina da Úniversidade de Såo Paulo na pessoa de seu diretor, Dr. Luiz Carlos Arcon, pelos dados fornecidos. 
MACHADO, A. de B.B. et al. [Human cysticercosis in a general hospital in S.Paulo, Brazil]. Rev. Saúde públ ., S.Paulo, 22: 240-4, 1988.

ABSTRACT: In this retrospective study of 126,968 in-patients at the Hospital das Clinicas of S. Paulo Medical School, S. Paulo, from 1979 to 1985 , cysticercosis was diagnosed in $260(0.2 \%)$ of them Epidemiological data obtained from these 260 patients showed a close distribution with regard to sex, and the age group from 20 to 50 years old was the most affected $(53.8 \%)$. Neurocysticercosis was the most frequent and the high rate $(91.5 \%)$ was understood to be due to the characteristics of specific the patients studied. Despite the recent development of the diagnostic and therapeutic technology, the average length of hospitalization was 35 days and the rate of lethality $14.6 \%$, pointing to the need for preventive measures on the part of public health programs.

UNITERMS: Cysticercosis, occurrence. Epidemiology. Hospitalization.

\section{REFERÊNCIAS BIBLIOGRÁFICAS}

1. BROTTO, W. Aspectos neurológicos da cisticercose. Arq. Neuro-psiq., S.Paulo, 5: 258-94, 1947.

2. CANELAS, H. M. Neurocisticercose: incidência, diagnóstico e formas clínicas. Arq. Nevro-psiquiat., S. Paulo, 20: 1-30, 1962.

3. COSTA, J. M.; FERREIRA, A. W.; MAKINO, M. M.; CAMARGO, M. E. Spinal fluid immunoenzimatic assay (ELISA) for neurocysticercosis. Rev. Inst. Med. trop. S. Paulo, 24: 337-41, 1982.

4. DAMONTE VICELLO, L. J. Desconecimiento de la epidemiología de la cisticercosis en México. Salud públ. Méx., 25: 301-5, 1983.

5. ESCALANTE, S. Epidemiología de la cisticercosis en el Peń. Rev. Neuro-psiquiat., 40: 29-51, 1977.

6. GOBBI, H.; ADAD, S. J.; NEVES, R. R.; ALMEIDA, H. O. Ocorrência de cisticercose (Cysticercus cellulosae) em pacientes necropsiados em Uberaba, MG. Rev. Pat. trop., 9: 51-9, 1980.

7. GOLDSMITH, R. S.; KAGAN, I. G.; REYES-GONZÁLEZ, M. A.; CEDENO FERREIRA, J. Estudios seroepidemiológicos realizados em Oaxaca, México. IEncuesta de anticuerpos parasitarios mediante la prueba de hemaglutinación indirecta. Bol. Ofic. sanit. Pa. numer., 71: 500-18, 1971.

8. GONZALES LUARCA, E. Situação atual do complexo teníase humana-cisticercose nas Américas. Comun. cient. Fac. Med. vet. Zootec. Univ. S. Paulo, 8: 223-6, 1984.

9. LANGE, O. Sindrome liquórica da cisticercose encéfalo-meningea. Rev. Neurol. Psiquiat. S. Paulo, 6: 35-48, 1940.

10. LIVRAMENTO, J. A. Contribuição de reaçōes de imunofluorescência no líquido cefalorraquiano ao estudo da neurocisticercose. Arq. Neuro-psiquiat., S. Paulo, 39:
$261-78,1981$

11. MACHADO, A. de B.B. Actual epidemiological data of neurocysticercosis in Såo Paulo. [Trabalho da Divisão do Laboratório Central do Hospital das Clínicas da Faculdade de Medicina da Universidade de São Paulo Mimeografado]

12. MAHAJAN, R. C. Geographical distribution of human cysticercosis. In: Flisser, A., ed. Cysticercasis: present state of knowledge and perspectives. New York, Academic Press, 1982. p. 39-46.

13. MATEOS, J. M.; BIAGI, F.; MARQUEZ-MONTER, H.; KRETSCHMER, R.; LÓPEZ-OSUNA, M.; SCHAAS, G. Cisticercosis cerebral como problema de salud pública. Gac. méd. Méx., 103: 225-50, 1972.

14. SARTI GUTIÉRREZ, E. J. \& GUTIERREZ OSPINA, I. La teniasis y cisticercosis en México (revisión bibliográfica): Salud. públ. Méx., 28: 556-63, 1986.

15. SCHENONE, H.; RAMIREZ, R.; ROJAS, A. Aspectos epidemiológicos de la neurocisticercosis en América Latina. Bd. chil. Parasit., 28: 61-72, 1973.

16. SCHENONE, H.; VELARROEL, F.; ROJAS, A.; RAMIREZ, R. Epidemiology of human cysticercosis in Latin America. In: Flisser, A., ed. Cysticercasis: present state of knowledge and perspectives. New York, Academic Press, 1982. p. 25-38.

17. SPDNA-FRANÇA, A. Cisticercose do sistema nervoso central: consideraçöes sobre 50 casos. Rev. paul. Med., 48: 59-70, 1956.

18. SPINA-FRANÇA, A. Cisticercose do sisteme nervoso central. In: Canelas, H.M., ed. Manual de clínica meurológica. Sto Paulo, Sarvier, 1967. p. 237-45.

19. SPINA-FRANÇA, A. Imunobiologia da cisticercose: avaliaçio dos conceitos atuais. Arq. Neuro-psiquiat. S. Paulo, 27: 125-40, 1969. 
20. TAKAYANAGHI, O.M. \& JARDIM, E. Aspectos clínicos da neurocisticercose. Arq. Neuro-psiquiat., S. Paulo, 41 : $50-63,1983$.

21. WOODHOUSE, E.; FLISSER, A.; LARRALDE, C. Seroepidemiology of human cysticercosis in Mexico. In: Flisser, A., ed. Cysticercosis: present state of knowl- edge and perspectives. New York, Academic Press , 1982. p. 11-23.

Recebido para publicação em 4/8/1987 Reapresentado em 12/11/1987 Aprovado para publicaçäo em 17/11/1987 